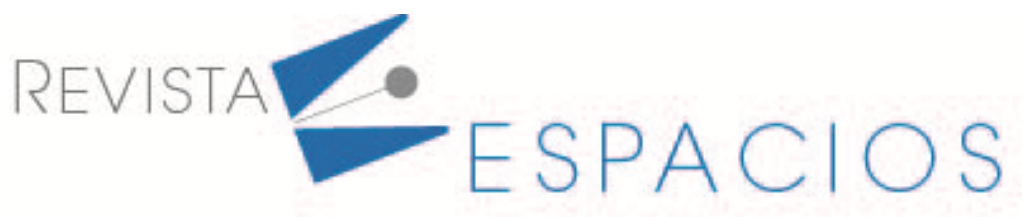

Vol. 41 (45) $2020 \cdot$ Art. 11

Recibido/Received: 21/07/2020 • Aprobado/Approved: 29/09/2020 • Publicado/Published: 26/11/2020

\title{
Evolución de la transferencia tecnológica a través del modelo Spin-off
}

\section{Evolution of technology transfer the Spin-off model}

\author{
AGUILLÓN, Marilce A. ${ }^{1}$ \\ ORDÚZ, Fabián ${ }^{2}$ \\ MARIÑO, Gladys Y. ${ }^{3}$
}

\begin{abstract}
Resumen
Se presenta el estado de la investigación científica en relación con el tema de transferencia tecnológica por medio de Spin-off. La Investigación de carácter documental utilizó como fuente de exploración bases de datos académicas, entre los años 1990 a 2019. La información se sistematiza en 3 categorías: conceptualización; evolución de la transferencia tecnológica en Norteamérica, Europa, América Latina y Colombia y finalmente; Spin-off. Como resultado, se confirma a las Spin-off como el mecanismo más efectivo de transferencia tecnológica.

Palabras clave: transferencia tecnológica, spin-off, gestión de la innovación, gestión del conocimiento
\end{abstract}

\begin{abstract}
The state of scientific research in relation to the topic of technology transfer through Spin-off is presented. Documentary research, used academic databases as a source of exploration, between the years 1990 to 2019. The information is systematized into 3 categories: conceptualization; evolution of technology transfer in North America, Europe, Latin America and Colombia and finally; Spin-off. As a result, Spin-offs are confirmed as the most effective technology transfer mechanism.

Keywords: Technology transfer, Spin-off, Innovation management, Knowledge management
\end{abstract}

\section{Introducción}

El conocimiento es el principal activo intangible de un individuo y por ende de la organización o sociedad a la que pertenece, una de las principales fuentes de conocimiento se encuentra en las Instituciones de Educación Superior (IES) quienes, a través de su ejercicio misional, que involucra las labores de docencia, investigación y extensión o proyección social, se comprometen a gestionarlo para contribuir al desarrollo de la sociedad. En este sentido, en cuanto a la docencia, las IES se comprometen a formar profesionales integrales competentes e idóneos; en investigación a desarrollar procesos investigativos que ayuden a comprender la realidad y a generar conocimiento; en extensión, a hacer transferencia de conocimiento para aplicarlo y lograr un mejoramiento del entorno.

\footnotetext{
${ }^{1}$ Maestrante en Administración de las organizaciones. Universidad Pedagógica y Tecnológica de Colombia. Colombia. Email: marilce.aguillon@uptc.edu.co

${ }^{2}$ Maestrante en Administración de las organizaciones. . Universidad Pedagógica y Tecnológica de Colombia. Colombia. Email: jeisson.fonseca@uptc.edu.co

${ }^{3}$ Magister en Administración. Docente investigadora Universidad Pedagógica y Tecnológica de Colombia. Colombia. Email: gladys.marino01@uptc.edu.co
} 
La transferencia de conocimiento aporta al desarrollo económico de los territorios, en el sentido que posibilita, entre otros: el mejoramiento de procesos, soluciones técnicas a problemas, diseño de nuevos materiales, productos y servicios que, sustentados en innovación y desarrollo tecnológico, mejoran la productividad y competitividad en los diferentes sectores de la economía.

La transferencia tecnológica es una forma de realizar transferencia de conocimiento, se entiende como la difusión de nuevo conocimiento, resultado de actividades de investigación obtenidas en las IES, para ser aplicado por la sociedad u otras instituciones. (Touriñán, 2019), así mismo el modelo Spin-off es un mecanismo para hacer transferencia tecnológica.

Spin-off hace referencia a nuevas empresas que nacen y se consolidan a partir del conocimiento surgido en la investigación de profesionales de una universidad o un centro de investigación; su finalidad es desplegar nuevos productos o servicios aterrizados al campo empresarial para que éstos sean utilizados con fines comerciales, generando beneficios para la sociedad, academia y empresa (López, 2017).

El objetivo de la investigación es realizar una revisión teórica sobre la transferencia tecnológica por medio del modelo Spin-off, se abordan como ejes temáticos relacionados el conocimiento, la gestión del conocimiento, la transferencia de conocimiento y la innovación. El análisis teórico se enfoca en identificar las tendencias, los factores determinantes y actores esenciales para la creación de empresas Spin-off.

Como conclusiones principales se encuentra que existen tres ramas tradicionales de transferencia de conocimiento: modelo lineal, dinámico e integrado así mismo, que el mayor foco de estudio hacia la transferencia de conocimiento por medio de empresas Spin-off son las desarrolladas por el modelo triple hélice. Dentro de los actores que deben intervenir para la creación de Spin-off están el gobierno y las universidades: el gobierno mediante apoyos financieros y definición de políticas públicas y las universidades a través de la transferencia tecnológica. Para el caso de las universidades es necesaria la existencia de una estrategia y estructura que permita una adecuada relación academia-estado. Por otra parte, dentro de los factores determinantes para la generación del modelo Spin-off, se encuentran el conocimiento y relación con el entorno; la innovación; la capacidad creativa; la investigación y el emprendimiento.

\section{Metodología}

\subsection{Tipo de investigación}

La investigación se realizó por medio de un análisis bibliográfico de tipo documental. Se plantea una descripción y análisis del estado de la investigación científica en relación con el tema de transferencia tecnológica por medio de Spin-off, teniendo como fuente de exploración las bases académicas: SCOPUS, WEB OF SCIENCE, ScienceDirect, Dialnet, SCIELO y Google Académico, durante el periodo de tiempo comprendido entre 1990-2019 para el marco teórico y los años 2005 hasta 2019 para la revisión sistemática.

El estudio busca profundizar sobre el tema de transferencia de conocimiento por medio de empresas Spin-off, los resultados se especifican en la construcción de un marco teórico, entendido como un "corpus de conceptos de diferente niveles de abstracción articulados entre sí que orientan la forma de aprehender la realidad" (Sautu, Boniolo, Dalle, y Elbert, 2005, p. 34), y el análisis de la evolución y desarrollo del temario recopilado en una revisión sistemática (conocido también como estado del arte), que es "una metodología de investigación cualitativo-documental de carácter crítico-interpretativa que revisa los estados producidos por las personas en su representación bibliográfica, conformada por tres fases: 1) planeación y diseño, 2) gestión y análisis, 3) formalización y elaboración" (Gómez, Galeano, y Jaramillo, 2015, p. 423). 


\subsection{Criterios de selección de los documentos}

Se tuvo como criterios de inclusión los siguientes aspectos:

1. Filtro por publicación: Se seleccionaron artículos resultado de investigación en las áreas de: ciencias sociales; ciencias humanas; educativas y económicas, que fueran publicados en el periodo comprendido entre 2005 y 2019 para la revisión sistemática y para el marco teórico entre 1990 y 2019.

2. Filtro por palabras: Se tomaron como palabras fijas claves: gestión del conocimiento; gestión de la innovación; modelo Spin-off; transferencia tecnológica y creación de empresas Spin-off.

3. Filtro por contenido objetivo y confiable: Se tuvo en cuenta el número de citaciones para evidenciar la credibilidad y aceptación del artículo en la comunidad científica, además que el artículo tuviera más de 50 referencias para tener información verídica y actualizada.

4. Criterios de exclusión: fueron excluidas las investigaciones anteriores al año 2004 para el caso de la revisión sistemática y al año 1990 para el caso de marco teórico.

\subsection{Selección de documentos}

Se realizó una preselección de doscientos veintitrés (223) artículos, sobre los cuales se aplicaron los criterios de selección de documentos dando como resultado la selección de cuarenta (40) artículos para la revisión sistemática y veintinueve (29) para el marco teórico, para un total de 69 artículos consultados (5 artículos se tuvieron en cuenta tanto en el marco teórico como en la revisión sistemática), de esta forma se consolida el estudio con sesenta y cuatro (64) artículos. La información se organizó y clasificó a través de una tabla de conocimiento que describe las variables de estudio. En la Figura 1 se describe el proceso de selección.

Figura 1

Prisma para la selección de artículos

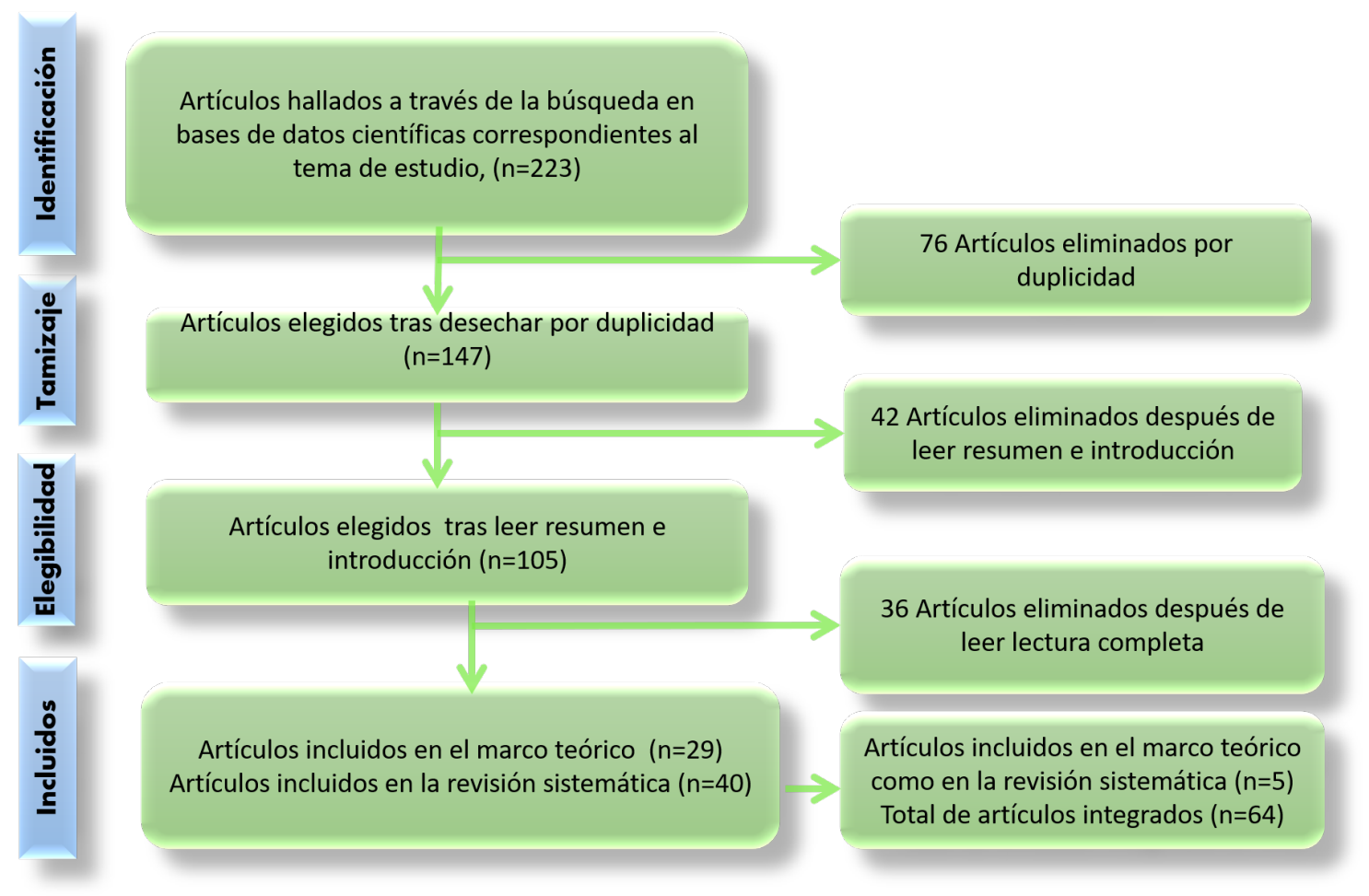

Fuente: Elaborado por los autores del presente estudio 


\subsection{Categorías de análisis}

Como resultado del análisis e identificación de los ejes temáticos de los artículos seleccionados, se aborda la información desde tres (3) categorías: conceptualización sobre gestión del conocimiento e innovación, transferencia tecnológica y su relación con las IES; Spin-off y; evolución y experiencias en transferencia tecnológica por medio de Spin-off en diferentes ámbitos geográficos (Norteamérica, Europa, América Latina y Colombia). El Cuadro 1 describe la relación de artículos por categoría y por tema.

Cuadro 1

Temas por categoría de análisis

\begin{tabular}{|c|c|c|c|}
\hline Objetivo & Categoría de análisis & Temática principal & N.o de artículos \\
\hline \multirow[t]{3}{*}{ MARCO TEÓRICO } & $\begin{array}{l}\text { Conceptualización sobre gestión del } \\
\text { conocimiento e innovación, } \\
\text { transferencia tecnológica y su } \\
\text { relación con las IES, Spin-off }\end{array}$ & $\begin{array}{l}\text { Conceptualización sobre transferencia } \\
\text { tecnológica, gestión innovación y Spin-off }\end{array}$ & 22 \\
\hline & \multirow[t]{2}{*}{ Spin-off } & Tipos de modelos Spin-off & 4 \\
\hline & & $\begin{array}{l}\text { Modelo de factores determinantes para la } \\
\text { creación de Spin-off académicas. }\end{array}$ & 1 \\
\hline \multirow{5}{*}{$\begin{array}{l}\text { REVISION } \\
\text { SISTEMÁTICA }\end{array}$} & \multirow{5}{*}{$\begin{array}{l}\text { Evolución y experiencias en } \\
\text { transferencia tecnológica por medio } \\
\text { de Spin-off en diferentes ámbitos } \\
\text { geográficos (Norteamérica, Europa, } \\
\text { América Latina, Colombia) }\end{array}$} & Relación con la triple hélice y factores de éxito & 2 \\
\hline & & $\begin{array}{l}\text { Primeras empresas Spin-off y acercamiento en } \\
\text { Norteamérica }\end{array}$ & 7 \\
\hline & & $\begin{array}{l}\text { Inicios e historia en Europa de transferencia } \\
\text { tecnológica Spin-off }\end{array}$ & 15 \\
\hline & & Transferencia tecnológica en América Latina & 10 \\
\hline & & $\begin{array}{l}\text { Literatura de transferencia tecnológica en } \\
\text { Colombia }\end{array}$ & 7 \\
\hline
\end{tabular}

Fuente: Elaborado por los autores del presente estudio

\section{Resultados}

\subsection{Marco teórico}

\subsubsection{Gestión del conocimiento y la innovación}

Se entiende al conocimiento como "un flujo en el que se mezclan la experiencia, valores importantes, información contextual y puntos de vista de expertos, que facilitan un marco de análisis para la evaluación e incorporación de nuevas experiencias e información (Segarra y Bou, 2005, p. 179), en este sentido, el conocimiento, a nivel individual y organizacional, debe gestionarse de manera eficiente, "tal como lo plantea Rodríguez (2002), genera la verdadera ventaja competitiva de una organización y de un país, ya que en esta reside su capacidad de gestionar sus procesos de creación, desarrollo y difusión del conocimiento" (Marulanda y López, 2013) p. 160. Por ende, es la adecuada gestión del conocimiento lo que lleva a una organización a explorar nuevos caminos por recorrer, traducido en la creación de nuevos bienes y servicios de alta utilidad para la sociedad, mejoras en productos antiguos, desarrollo de nuevos sistemas de gestión para la organización y el fortalecimiento de los convenios entre organizaciones (Nonaka, 1994; Teece, Pisano y Shuen, 1997; Alavy y Leidner, 2001).

Es importante tener en cuenta que el término "conocimiento" abarca mucho más que tan solo una función específica dada por el aprendizaje recibido en las IES, pues el significado de éste es mucho más amplio y general. Por otra parte, la tecnología es el producto del conocimiento enfocado en alguna de las ramas de la investigación, término que ya es más discreto y directo para su definición en un campo de la ciencia (Nonaka, 1994; Touriñán, 2019).

“(...) me gustaría aclarar que en el uso normal del lenguaje se dice transferencia de tecnología, transferencia de resultados y sin embargo hay una cierta tendencia a decir transferencia del 
conocimiento. Lo cierto es que hay más conocimiento del que se transfiere y, por tanto, yo usaré la preposición y no la contracción, porque realmente se transfiere algún conocimiento, no el conocimiento". (Touriñán, 2019)

El conocimiento es uno de los resultados que se genera a través de las actividades de formación y de investigación que se adelantan en las Instituciones de Educación Superior, Sin embargo, este conocimiento, no siempre se gestiona hacia su transferencia de manera efectiva para ser aplicado por la sociedad, queda limitado a registrarse en documentos como artículos, informes técnicos y ponencias, entre otros, lo que no garantiza su aplicación real. Una efectiva transferencia tecnológica a la sociedad desde las IES podría contribuir, entre otros, a la creación y mejoramiento de empresas, disposición de nuevas oportunidades y alternativas de inversión y empleo y en consecuencia, elevar el nivel de competitividad del territorio. De otra parte, las IES, a través de la transferencia tecnológica, deben aportar para transformar el contexto en el cual el nivel de desempleo al que se enfrentan las personas recién graduadas no les permite desempeñarse en trabajos que requieran aplicar las competencias adquiridas en su proceso de aprendizaje, por lo que terminan haciendo labores que pueden llegar a ser muy distintas para las que fueron capacitadas, en último término, la desaparición del conocimiento adquirido. Razón por la que es importante que las IES no desaprovechen el conocimiento impartido y lo transfieran a la sociedad, en un alto grado de pertinencia (Stankiewicz, 1994; Miller, McAdam, Moffett y Brennan, 2011).

Entonces, el uso efectivo de la tecnología que genera una organización, para este caso el producido por las IES, dinamiza procesos de innovación, innovación tomada como factor de éxito que es resultado de la aplicación de una correcta estrategia de mercado fruto de un adecuado uso de la tecnología, en otras palabras, lo que se conoce como gestión de la innovación. Meisel, Bermeo, y Oviedo (2006) plantean:

"La gestión de la innovación (GI), implica la puesta en marcha de acciones que conlleven a la introducción exitosa al mercado de nuevas ideas o invenciones, representadas en productos, procesos o formas de operar (mercadeo, organización o comercialización). La GI supone un proceso complejo y dinámico en el que permanentemente se incorpora, apropia y transforma conocimiento científico y técnico existente al interior (empleados y tecnología instalada) y exterior (comunidad científica, proveedores, competidores) de la organización" p. 166.

\subsubsection{Transferencia tecnológica y su relación con las IES}

La transferencia tecnológica se fundamenta en la relación entre la universidad y la industria que conduce a aportar resultados de investigación sujetos de actividad comercial, se materializa cuando se presenta un producto (artefactos, patentes, licencias, etc.) fruto de la academia y responde a la necesidad específica de una industria en particular (Geuna y Muscio, 2009; Martínez-Gómez, 2017). En este sentido, los gobiernos fomentan activamente la iniciativa de transferencia tecnológica mediante universidad-industria, pues tienen el potencial de impulsar el crecimiento económico (Bercovitz y Feldman, 2006 citado por Robertson , McCarthy y Pitt, 2019) p. 4, (Etzkowitz y Peters, 1990; Etzkowitz, 1996).

También se vincula la relación que puede tener la transferencia tecnológica con el impacto de las ciencias sociales, pues esta transferencia es distinta, ya que en ella escasean los productos tangibles del conocimiento como patentes o licencias, pero en cambio, se puede generar innovación social. Esta relación se manifiesta de manera directa e indirecta, de manera directa a través de publicaciones como libros, artículos sociales y manuales; y de manera indirecta, por medio de consultorías e investigaciones por contrato (Etzkowitz, 1996; Huyghe, Knockaert, Wright y Piva, 2014; Olmos-Peñuela, Castro-Martinez y D'este, 2014). De otra parte, la transferencia tecnológica, a través de la relación universidad-industria, aporta a la comprensión y generación de cambios del componente social dentro de las organizaciones, pues permite impactar en el quehacer y el valor de quiénes y cómo interactúan las personas (Robertson, McCarthy y Pitt, 2019). 
La transferencia tecnológica es el conjunto de actividades dirigidas a la difusión de conocimientos, experiencia y habilidades con el fin de facilitar el uso, la aplicación y la explotación del conocimiento y las capacidades en I+D de la universidad fuera del ámbito académico, ya sea por otras instituciones de I+D, el sector productivo o la sociedad en general (Touriñán, 2019; Etzkowitz \& Peters, 1990). En este sentido, el uso del conocimiento generado en las IES puede tomar dos rutas: la sociedad y lo que ella demanda para su bienestar y el sector productivo.

Para cumplir con el objetivo de llevar el conocimiento fuera del ámbito académico, y en particular, orientado al sector productivo, se pueden identificar las siguientes formas:

1. Modelo lineal, en el cual la transferencia está precedida de los descubrimientos científicos y de la consecución de patentes. En el modelo lineal están implicados la universidad, el científico o centro de investigación, la oficina de transferencia de resultados de investigación (OTRI) y las empresas (MartínezGómez, 2017).

2. Modelo dinámico, que mejora el modelo lineal y tiene como propósito la transferencia tecnológica por medio de la comercialización o difusión (Martínez-Gómez, 2017).

3. Modelo integrado, que ha evolucionado, desde el protagonismo del Estado en las relaciones entre las empresas y el Estado, a una interacción entre el Estado, la empresa y la universidad con campos de acción delimitados para cada uno; para llegar a su versión última, en la que cada uno de los actores asume las funciones propias y las de todos, de tal manera que las universidades crean empresas y éstas, a su vez, crean centros de investigación (Martínez-Gómez, 2017).

\subsection{Empresa de base tecnológica Spin-off}

Las empresas de base tecnológica son llamadas Spin-off, que nacen en el corazón de la IES, con un alto componente en investigación y que dirigen su producción al sector privado y comercial. La Spin-off es el método más común utilizado por las IES para hacer transferencia tecnológica (Stankiewicz, 1994; Etzkowitz y Leydesdorff, 2000). La puesta en marcha de proyectos empresariales (Spin-off) es un mecanismo que las universidades pueden utilizar para transferir resultados de la investigación pública en el sistema económico (Castillo y Alvarez, 2015). La concepción de Spin-off se remonta al siglo pasado cuando se teoriza la relación entre GobiernoUniversidad-Industria (GUI), conocido como el modelo de triple hélice.

\subsubsection{El modelo de triple hélice}

La relaciones Gobierno-Universidad-Industria proporcionan una metodología óptima para el emprendimiento y la innovación, trasladando la investigación/conocimiento a la práctica/uso (Etzkowitz y Zhou, 2018). La interacción GUI es un enfoque sociológico para el análisis de la innovación (Etzkowitz, 1994, Leydesdorffy Etzkowitz, 1996 citado en González de la Fe, 2009). Etzkowitz (2002), afirma que los nuevos arreglos sociales y canales de interacción son necesarios si la industria y el gobierno están unidos por las universidades en economías basadas en el conocimiento (citado en Chang, 2010). En el siglo XXI se cuenta con una interpretación más concreta del modelo, realizada por Chang, 2010, donde "se conoce como un proceso intelectual orientado a visualizar la evolución de las relaciones entre universidad-sociedad, y por otro lado caracterizado por la intervención de la universidad en los procesos económicos y sociales" p. 86.

La relación GUI es una fuente para la creación de nuevas empresas de base tecnológica con alto grado de innovación. Las tres palas de la hélice funcionan de tal manera que, la universidad cumple con el potencial para el conocimiento innovador, la industria cumple con el anclaje a las posibilidades del mercado y el gobierno, con 
recursos económicos, normas e incentivos de las políticas públicas de innovación (González de la Fe, 2009; (Etzkowitz y Leydesdorff, 1995; Etzkowitz y Leydesdorff, 1996; Etzkowitz y Leydesdorff, 2000).

De este modo, del modelo triple hélice surge la creación de empresas que están basadas en conocimiento, nacidas en el corazón de la universidad y con salida al sector industrial. El término Spin-off corresponde a estas empresas creadas del modelo de triple hélice, que se puede definir mejor como una "escisión", entendido como la división de una organización en 2 o más sociedades ya sean nuevas o preexistentes, para el caso de la Spin-off, es una empresa nacida en la universidad que toma rumbo hacia la industrial y hacía el gobierno (Etzkowitz y Leydesdorff, 1995; Giha, Olaya, Zapata, Pontón, Rojas, Franco, López, Mejía, Trujillo y Betancur, 2016).

Los estudios que abarcan al modelo de triple hélice han puesto su interés en el papel de la sociedad civil, como un cuarto agente del modelo, pues la relación tradicional de Gobierno-Universidad-Industria refleja su impacto en la sociedad. "Las hélices representan la especialización y la codificación en los sistemas funcionales que evolucionan a partir y en el seno de la sociedad civil" (Leydesdorff, 2012, p. 30.) Se entiende que la sociedad civil, siendo la cuarta hélice, funciona como agente receptor del impacto fruto de la relación tradicional de GobiernoUniversidad-Industria, pero no necesariamente está directamente vinculada al modelo de triple hélice, pues se pone en consideración los criterios en los que se construye el modelo de triple hélice, tales como financiación, enlace institucional y modelo de operación (Sierra, 2018).

\subsubsection{Tipologías de Spin-off}

La organización Spin-off puede abarcar múltiples realidades, según (Beraza y Rodríguez, 2012), la Spin-off se puede categorizar en 4 tipologías:

Spin-off Independiente: Son las empresas creadas por iniciativa propia del investigador, que no son necesariamente demasiado rígidas, lo que les da libertad de escoger su forma de financiamiento y su cronograma de actividades, y además, no es necesario en alto nivel, incluso inexistente, la ayuda financiera de entidades externas. Se caracteriza porque miembros de la comunidad universitaria crean una nueva empresa para explotar comercialmente el conocimiento acumulado durante sus actividades académicas (conocimiento tácito).

Spin-off Vinculada: en este caso, los miembros de la comunidad científica con espíritu emprendedor o la propia universidad promueven la creación de una nueva empresa para explotar comercialmente los resultados de investigación desarrollada en el seno de la universidad.

Join Ventures: La universidad, como institución, impulsa la creación de una nueva empresa para explotar comercialmente una tecnología con grandes perspectivas de futuro que espera le reporte recursos financieros externos. Para lograrlo, la universidad establece una join venture con otra $u$ otras organizaciones que aporten los recursos complementarios necesarios para el éxito de la empresa.

Spin-off Subsidiaria: en este caso la universidad, como institución, busca un socio industrial que aporte recursos para el desarrollo y comercialización de una nueva tecnología, o al contrario, un socio industrial busca una tecnología universitaria para su desarrollo y comercialización. En este caso, la nueva empresa es creada por otra ya existente como subsidiaria de ésta, y la universidad se limita a conceder una licencia de explotación de la tecnología a la nueva empresa.

Las diferentes formas en cómo se crea, financia y establece una Spin-off puede obedecer a alguna de las cuatro corrientes descritas anteriormente, que funciona como un punto de partida epistemológico para su creación. El Cuadro 2 presenta la tipología de Spin-offs académicas desde dos enfoques: enlace institucional y modelo de negocio. El enfoque de enlace institucional se refiere al cómo se crea y conforma una Spin-off y el enfoque modelo de negocio, a la manera como va a operar dicha empresa (Gómez, Mira y Verdú, 2007). 


\section{Cuadro 2}

Tipologías de spin-offs académicas según enfoques de análisis

\begin{tabular}{|c|c|c|}
\hline Perspectiva Y Enfoques & Tipología de Spin-off & Estudios \\
\hline \multicolumn{3}{|c|}{ Enlace Institucional } \\
\hline \multirow{3}{*}{$\begin{array}{l}\text { Dependencia/formalidad de la } \\
\text { tecnología transferida. }\end{array}$} & Spin-offs con dependencia directa & \multirow{6}{*}{$\begin{array}{l}\text { Roberts (1991); Pirnay et al. (2003); Hindle } \\
\text { and Yencken, (2004) Steffensen et al., } \\
\text { (2000); DeGroof and Roberts (2004) } \\
\text { Radosevich, (1995); Franklin et al. 2001; } \\
\text { Nicolaou and Birley, (2003); Pirnay et al., } \\
\text { (2003). }\end{array}$} \\
\hline & Spin-offs con dependencia parcial & \\
\hline & Spin-offs planificadas & \\
\hline \multirow[t]{2}{*}{ Compromiso de la universidad } & Spin-offs espontaneas & \\
\hline & Interno (personal investigador) & \\
\hline $\begin{array}{l}\text { Status/procedencia } \\
\text { emprendedor }\end{array}$ & Externo & \\
\hline \multicolumn{3}{|c|}{ Modelo de negocio } \\
\hline \multirow{6}{*}{$\begin{array}{l}\text { Actividad desarrollada } \\
\text { Transformación de la tecnología en } \\
\text { valor comercial }\end{array}$} & Spin-offs orientadas al mercado & \multirow{6}{*}{$\begin{array}{l}\text { Stankiewicz, (1994); Chiesa and Piccaluga, } \\
\text { (2000); Bower, (2003); Pirnay et al., (2003) } \\
\text { Mustar et al. (2006); Branscomb and } \\
\text { Mustar et al. (2006); Branscomb and } \\
\text { Clarysse, (2004); Druilhe and Garnsey, } \\
\text { (2004); Aggarwal and Bayus (2002) }\end{array}$} \\
\hline & $\begin{array}{l}\text { Spin-offs orientadas al desarrollo de } \\
\text { activos tecnológicos. }\end{array}$ & \\
\hline & Spin-offs plataforma & \\
\hline & Spin-offs producto & \\
\hline & Spin-offs plataforma-producto & \\
\hline & Spin-offs prospectaras. & \\
\hline
\end{tabular}

Fuente: (Gómez, et al., 2007)

\subsubsection{Modelo de factores determinantes para la creación de Spin-off académicas.}

Dentro de los modelos para generar empresas Spin-off se encuentra el modelo de Monge-A, Briones-P, y GarcíaP. (2011), en el cual se plantean los factores determinantes, como se ilustra en la Figura 2, se evidencian cuatro factores, el primero de ellos son los atributos y las características de personalidad de los emprendedores académicos; el segundo, los recursos propios y las capacidades de la universidad, tercero; la estructura universitaria y las políticas de comercialización y cuarto; los factores ambientales que influyen en los emprendimientos académicos.

Figura 2

Modelo de factores determinantes para la creación de Spin-off académicas.

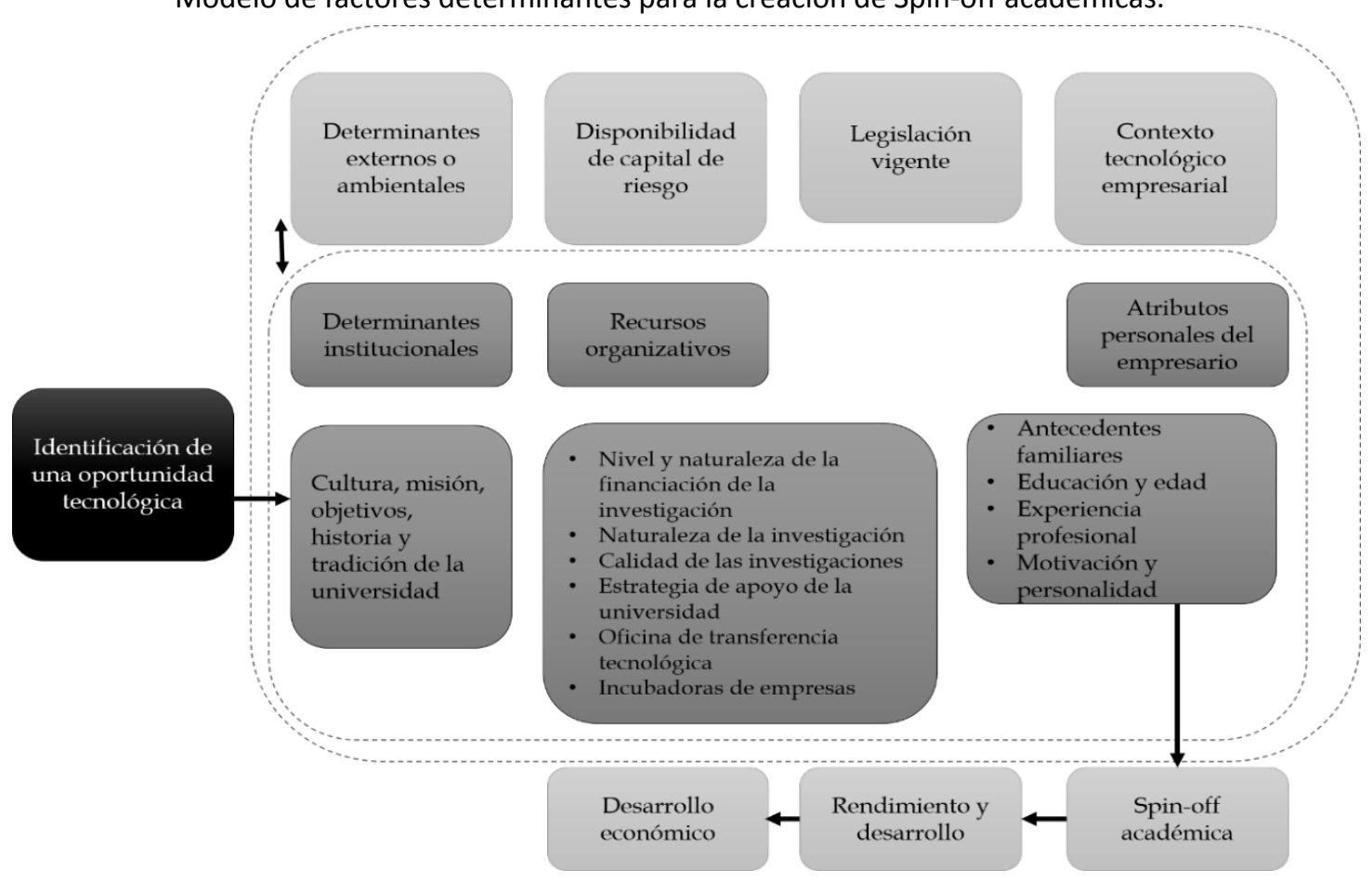

Fuente: Monge-A, et al., (2011) 


\subsection{Revisión sistemática}

Si bien en Colombia se denota variedad en investigación en Spin-off, tiene aristas en su aplicabilidad, puesto que existen pocas empresas Spin-off universitarias en Colombia, por eso es fundamental realizar investigación a nivel internacional, para generar literatura relevante, oportuna y así identificar los factores y actores determinantes que se tuvieron en cuenta para formar empresas Spin-off y que se puedan mantener en el tiempo. Se evidencia que en el campo de la gestión de la innovación y el conocimiento por medio de la transferencia tecnológica se ha generado un mayor impacto en países tales como Reino Unido, Suecia, Canadá, Estados Unidos y Francia, estos países han ayudado a la academia a relacionarse con el sector empresarial, por la forma que se han vinculado, caso contrario se ve en América Latina que aunque se evidencia diversas publicaciones en relación con transferencia tecnológica por medio de Spin-off, ha tenido pocos avances en su aplicabilidad (ver Cuadro 3).

Cuadro 3

Número de artículos publicados por país

\begin{tabular}{|c|c|c|c|c|c|}
\hline País & $\begin{array}{c}\text { N.o de } \\
\text { artículos }\end{array}$ & País & $\begin{array}{c}\text { N.o de } \\
\text { artículos }\end{array}$ & País & $\begin{array}{c}\text { N.o de } \\
\text { artículos }\end{array}$ \\
\hline Colombia & 7 & Reino Unido & 4 & México & 2 \\
\hline España & 5 & Brasil & 3 & Argentina & 1 \\
\hline Italia & 5 & Canadá & 3 & Venezuela & 1 \\
\hline Estados Unidos & 4 & Costa Rica & 3 & Holanda & 1 \\
\hline
\end{tabular}

Fuente: Elaborado por los autores del presente estudio

\subsubsection{Transferencia tecnológica por medio de Spin-off en Norteamérica}

Dentro de la corriente de la gestión de la innovación (GI) es necesario que se lleve al mercado acciones con buenas y nuevas ideas, que estén dirigidas a productos, procesos y/o formas de operar, proceso que requiere de celeridad para afrontar los continuos cambios del entorno. Esta dinámica se alimenta de manera permanente con la incorporación, apropiación y transformación de conocimiento tanto fuera como dentro de las organizaciones (Meisel, et al., 2006). Una de las formas de hacer que la gestión de la innovación cumpla su objetivo es por medio de la transferencia tecnológica con el modelo Spin-off. Un país que quiera promover la creación de empresas Spin-off perdurables en el tiempo, debe tener en cuenta las regulaciones gubernamentales, políticas internas del sector al que pertenecen, características del contexto y los mecanismos necesarios para apoyar no sólo su creación sino su sostenibilidad. Por ende, cuando se tienen entornos altamente emprendedores las políticas más adecuadas son de baja selectividad y bajo soporte, ya que se puede escoger excelentes emprendimientos a bajo costo, mientras que entornos subdesarrollados, se requieren de alta selectividad y alto apoyo, puesto que en se debe escoger a la de mayor impacto positivo y con mayor potencial para aumentar la posibilidad de crear Spin-off estables y ganadoras. (Breznitz, O'Shea, Allen, 2008). En las investigaciones revisadas se expresa que factores tienen en cuenta los diferentes países para formar una Spinoff y que sea exitosa (ver Cuadro 4). 


\section{Cuadro 4}

Estudios relacionados con la correlación de la triple o cuarta Hélice y factores que consideran determinantes para crear Spin-off y ser exitosas.

\begin{tabular}{|c|c|c|}
\hline País & $\begin{array}{l}\text { Artículos } \\
\text { relacionados } \\
\text { con la triple } \\
\text { o cuarta } \\
\text { Hélice }\end{array}$ & Factores determinantes en cada país para ser exitosos \\
\hline Colombia & 2 & $\begin{array}{l}\text { Financiación gubernamental, entes internos de la universidad, actividades de investigación } \\
\text { y extensión }\end{array}$ \\
\hline España & 2 & $\begin{array}{l}\text { políticas científicas y tecnológicas para mejorar la cooperación que debe existir entre } \\
\text { universidad e industria, Colaboración universidad-empresa }\end{array}$ \\
\hline Brasil & - & $\begin{array}{l}\text { Obtener licencias de patentes, desarrollar proyectos de investigación y desarrollo }(I+D) \text { que } \\
\text { fortalezcan el hilo empresarial, asociación para la creación de empresas derivadas }\end{array}$ \\
\hline Canadá & 1 & $\begin{array}{l}\text { Apoyo gubernamental, actitud emprendedora, investigadores disciplinados, profesorado } \\
\text { preparado, colaboración empresa Universidad }\end{array}$ \\
\hline Costa Rica & 1 & $\begin{array}{l}\text { Estudiar el entorno donde se encuentra, motivación del personal, para generar innovación y } \\
\text { conocimiento, ambiente laboral adecuado, fomentar la creatividad para asumir mejor los } \\
\text { riesgos, que los investigadores y empleados participen en cursos de formación. }\end{array}$ \\
\hline $\begin{array}{l}\text { Estados } \\
\text { Unidos }\end{array}$ & 2 & $\begin{array}{l}\text { Políticas que promuevan la creación de Spin-off, universidades colaborativas con las } \\
\text { organizaciones, capital institucional, financiación interna y externa, capital comercial y } \\
\text { humano, resultados derivados de la universidad por medio de la investigación se crean las } \\
\text { Spin-off, dependencia de la historia. }\end{array}$ \\
\hline México & - & $\begin{array}{l}\text { Investigación, Desarrollo e innovación. Inversión y financiación. Conocimiento científico y de } \\
\text { aprendizaje }\end{array}$ \\
\hline $\begin{array}{l}\text { Reino } \\
\text { Unido }\end{array}$ & 2 & $\begin{array}{l}\text { Conocimiento tecnológico específico, entrelazar universidad-empresa, apoyo } \\
\text { gubernamental }\end{array}$ \\
\hline Argentina & - & $\begin{array}{l}\text { Oficinas de Vinculación Tecnológica y Transferencia, presupuestos por parte del gobierno } \\
\text { para el conocimiento y la organización }\end{array}$ \\
\hline Chile & 1 & $\begin{array}{l}\text { Apoyo Gubernamental, actitud creadora e innovadora, fortalecimiento de vinculación } \\
\text { empresa-universidad }\end{array}$ \\
\hline Holanda & - & $\begin{array}{l}\text { Vinculación con el entorno, inversión en investigación y desarrollo de las universidades, } \\
\text { patentes. }\end{array}$ \\
\hline Italia & 1 & $\begin{array}{l}\text { Vinculación empresa universidad, complementariedad universidad-empresa, innovar } \\
\text { fabricar y distribuir en mercados favorables. }\end{array}$ \\
\hline Venezuela & - & $\begin{array}{l}\text { Vinculación entre los egresados, profesores y estudiantes, para tener emprenderismo en la } \\
\text { creación de Spin-off }\end{array}$ \\
\hline
\end{tabular}

Fuente: Elaborado por los autores del presente estudio

Los primeros acercamientos en la creación de empresas Spin-off se dieron en Estados Unidos en la mitad del siglo XX, producto de procesos de investigación diseñados y elaborados en California y Massachusetts, como se evidencia en los proyectos de Valle de Silicion y la Ruta 128, momento histórico en el cual Estados Unidos fomenta el desarrollo económico a partir de la industria de dispositivos eléctricos, aeronaves y tecnología emergente. Gracias a esta iniciativa se crean instituciones que ayudan a las universidades a fortalecer los laboratorios científico- investigativo (Gupte, 2007).

Las investigaciones en Estados Unidos acerca de Spin-off han contribuido en gran parte a la literatura sobre el tema a nivel mundial, al igual que en otros países se encuentra que la evolución de la creación de Spin-off, ha forjado un cambio positivo en la generación de empresas, contribuyendo a que las investigaciones realizadas en las universidades, no solo se queden allí, sino que haya transferencia hacia las empresas. El éxito y sostenibilidad de las Spin-off se basa en el conocimiento de la trayectoria de la Spin-off a través del tiempo (O'shea, Allen, Chevalier y Roche, 2005; Etzkowitz, 2013). En consecuencia, la estructura y organización de las universidades son aspectos fundamentales para hacer posible la fundamentación, creación y comportamiento de las Spin-off, 
la cual debe propender por el afianzamiento de una cultura emprendedora e innovadora para la comercialización de investigación, contar con apoyo financiero, por medio de becas, industria y apoyo gubernamental. Gracias a las Spin-off se logra mayor aprendizaje y acumulación de conocimiento tecnología (O'shea, et al., 2005).

Castillo y Álvarez (2015), comentan que Estados Unidos, por medio de la ley Bayh-Dole, regula la transferencia tecnológica universitaria, promueve la generación de valor, el desarrollo económico y comercialización del conocimiento que estimula la investigación realizada en cada una de las universidades. De este modo Estados Unidos logró que sus principales universidades formaran una oficina enfocada exclusivamente a la transferencia tecnológica, centrándose en las patentes y licencia de resultados.

En Canadá, pasa algo similar a Estados Unidos, la transferencia tecnológica ha sido un éxito por medio de las Spin-off, como lo manifiesta Rasmussen (2008), gracias a la política gubernamental implementada en el año 2002 que enfatiza en fuentes de financiación que apoyan el surgimiento de Spin-off, por medio de las investigaciones realizadas en las universidades con programas provinciales y federales. En este sentido, es un factor determinante el papel de los actores e instituciones que fomentan la comercialización de la investigación desarrollada en la academia, como estrategia de colaboración industria-universidad (Hanel y St-Pierre, 2006). Las universidades canadienses se deben integrar con las empresas que ayudan económicamente a la creación de Spin-off de esta forma, se consolidan Spin-off universitarias colaborativas con el sector empresarial, para que no haya competencia si no que mutuamente ayuden al crecimiento de un sector, de esta manera se logra crear con mayor proporción activos de propiedad intelectual (Landry, Amara y Rherrad, 2006).

\subsubsection{Transferencia tecnológica por medio de Spin-off en Europa}

En Europa la transferencia tecnológica se desarrolló gracias a los trabajos realizados por Etzkowitz en el año 1998, los cuales hacen un análisis de la relación y colaboración que existe entre universidad y empresa, lo que se conoce como universidades emprendedoras. Con fundamento en estos escritos, Europa vio la necesidad de la generación de la triple hélice, para reunir universidades, empresas y gobierno en una sola esfera, con el objetivo de fomentar el desarrollo social y económico y creación de empresas fomentadas en el conocimiento de las universidades (i Tarrés, 2008).

España y Portugal se caracterizaban por tener una baja cultura empresarial, escasa actitud emprendedora, bajo apoyo financiero para la comercialización de investigaciones universitarias, altos niveles de burocracia en las universidades y deficiente vinculación empresa-universidad, lo que provocó que tuvieran baja participación en transferencia tecnológica (Rodeiro-Pazos, Fernández-López, Otero-González y Rodríguez-Sandiáz, 2012). Este proceso ha cambiado en los últimos años, tal como lo expresa Fernández-López, Pérez-Astray, Rodeiro-Pazos y Calvo, (2015); Touriñán (2019); Valmaseda y Hernández (2012), quienes afirman que ha retornado a España la confianza en la colaboración universidad-empresa, que se visualiza la importancia de que la transferencia tecnológica se haga de acuerdo con el modelo de la triple hélice de tal forma que conlleve a la creación de empresas Spin-off universitarias, y que esté mediado por políticas científicas y tecnológicas que ayudan a mejorar la cooperación que debe existir entre universidad e industria. En el caso de Portugal, se creó una Red emergente de tecnología universitaria con políticas acordes para mejorar el clima empresarial entre universidad-empresa, negocios y científicos e investigadores de las universidades

En Italia, producto de las investigaciones realizadas por Fini, Grimaldi, Santoni y Sobrero (2011); Visintin y Pittino (2013) y Algieri, Aquino y Succurro (2013) se establece que las Spin-off deben ser complementarias, que ayuden al crecimiento y desarrollo de una región y/o país, con innovación, fabricación y distribución de productos o servicios, donde se estimula la innovación, se logra mayor competitividad, lo que logra mercados laborales favorables. Para ello, es esencial que las universidades posean oficinas, profesorado, investigadores, incubadoras, que de manera coordinada, ayuden a alcanzar el éxito del funcionamiento de una Spin-off, donde se debe integrar tanto personal académico con el no académico, para que exista un equilibrio de sus 
conocimientos científicos y experiencia. Geuna y Muscio (2009) y Geuna y Rossi (2011) afirman que la transferencia tecnológica es una estrategia para generar desarrollo económico, es necesario tener en cuenta el financiamiento para fortalecer las empresas, crear valor con condiciones estructurales eficientes para que haya innovación, esta estrategia se evidencia en Italia como a nivel de Europa, donde se han aumentado significativamente las patentes de propiedad universitaria.

En esta misma línea, el Reino Unido se encuentra en las fases iniciales, se plantea que el contexto donde se crea la Spin-off ayuda a desarrollar competencias tanto externas como internas para abrir caminos competitivos y emprendedores que facilitan la comercialización del conocimiento de manera más eficiente (Clarysse, Wright y Van de Velde, 2011; Rasmussen, Mosey y Wright, 2013a; Rasmussen, Mosey y Wright, 2013b). De acuerdo a Lawton-Smith y Ho (2006), se afirma que en la región de Oxfordshire tienen más nivel de supervivencia las empresas Spin-off que las empresas británicas, con un porcentaje del $90 \%$ Spin-off comparado con un $62 \%$ de las empresas británicas, igualmente se puede hablar de Francia, puesto que cuenta con un $84 \%$ de superveniencia de las Spin-off.

De acuerdo con la literatura investigada, hasta el momento se puede afirmar que este fenómeno se ha desarrollado con más fuerza en Estados Unidos, allí se ha consolidado y avanzado de tal forma que es considerado como líder mundial de transferencia tecnológica con el modelo Spin-off. Gracias a la legislación desarrollada en Estados Unidos se despliega un ambiente propicio que ayuda a que las universidades líderes en investigación se vinculen con el entorno de forma exitosa (Morales, Pineda, y Ávila, 2012; Jiménez, Maculan, Otálora, Moreira y Castellanos, 2013). Dentro de los casos que confirman esta afirmación están: el Instituto Tecnológico de Massachusset Institute of Technology (MIT), donde se identifican más de 4000 Spin-off y el de la Universidad de Stanford, son ejemplos reales del empleo y desarrollo de esta modalidad de transferencia de conocimiento (Castillo y Alvarez, 2015; Lockett, Siegel, Wright y Ensley, 2005; Fong-Reynoso, 2010).

\subsubsection{Transferencia tecnológica por medio de Spin-off en América Latina}

Esta tendencia de investigación relacionada con la transferencia tecnológica por medio de Spin-off se evidencia también en América, donde se ha constatado la presencia de Spin-off en diferentes países, como es el caso de Costa Rica, México, Argentina, Colombia, dando grandes cambios a la competitividad de las organizaciones gracias a la gestión de la innovación, pero una de las preguntas que surgen, es ¿cómo ha sido el desarrollo de las Spin-off en estos países, cuál ha sido su impacto? (ver Cuadro 5)

Se evidencia que la regulación para la creación de Spin-off es débil en Latinoamérica tal como lo indica Dias y Porto (2014), por lo que se considera como un desincentivo para la formación de este tipo de empresas y puede crear conflictos de interés entre los académicos e investigadores. Otras investigaciones afirman que dentro de los países destacados por el éxito competitivo de las Spin-off son las universidades públicas costarricenses, donde se considera que una Spin-off es exitosa dependiendo de la situación y/o entorno en que se encuentre, motivación del personal eje fundamental para generar investigación y conocimiento (Helm y Mauroner, 2007).

En una investigación a 44 Spin-off de universidades públicas costarricenses, en el año 2017, se encontró que los factores de éxito del modelo Spin-off son: disponer de un ambiente laboral democrático, libertad de pensamiento para fomentar la creatividad, asumir mayores riesgos, que los empleados participen en cursos de formación, generar nuevas ideas, proyectos, productos y servicios que satisfagan y se adapten a las necesidades de las personas. Sus fundadores tienen una mejor visión comercial, mayor capacidad de investigar y actualizar, que aquellas organizaciones que no cuentan con Spin-off. Los emprendedores académicos tienen mayor éxito empresarial cuando al salir al mercado laboral, poseen motivación en emprender y construir su propia empresa para poner en práctica todos los conocimientos técnicos adquiridos (Monge-A, 2018; Monge-A Briones-Peñalver y Lema, 2015). 
En México en la "Propuesta de un modelo de transferencia de conocimiento científico-Tecnológico para México" publicado por Feria (2009), afirma que el origen y sector donde se encuentran los centros de investigación juegan un papel importante en la creación de Spin-off, es indispensable que el conocimiento científico, la innovación, los sistemas nacionales de innovación (SIN) y la transferencia de tecnología tengan una estrecha relación para que puedan funcionar correctamente, ya que al existir diferentes tipos de conocimiento y de aprendizaje hace que en las organizaciones la gestión del conocimiento-tecnológico sea un proceso complejo, es decir es dinámica y colectiva. Existe una gran preocupación porque en México no existe de manera formal un sistema nacional de innovación (SIN) lo cual lo hace frágil, por lo anterior la interacción entre diferentes actores y tecnología es escasa, pero a pesar de esto, se destacan organizaciones maduras y con buena trayectoria que se consolidan con la aplicación de diversos enfoques de gestión a través de Investigación y desarrollo (Feria, 2009).

En Argentina, se manejan interfaz entre los sectores científico-tecnológicos y los productivos, las cuales son denominadas "Oficinas de Vinculación Tecnológica y Transferencia", tuvo sus inicios aproximadamente hace 25 años, pero su comportamiento y evolución han sido impares, aunque el gobierno en los últimos años viene desarrollando incrementos presupuestarios para ayudar a fortalecer y afianzar la relación entre los actores del sistema nacional de innovación. Las universidades argentinas son fundamentales para la generación de conocimiento por medio de los grupos de investigación que tengan reconocimiento internacional (Malizia, Sánchez, Lombera y Castro, 2013).

En Brasil, la transferencia tecnológica no ha sido constante debido a la carencia de políticas institucionales y a las restricciones existentes de personal en las agencias de innovación, que ayudan a la formación de empresas Spin-off. Con el paso del tiempo, estas carencias se han venido debilitando por medio del fortalecimiento de la institución de innovación con el desarrollo tecnológico, de otra parte, tal como lo describe Dias y Porto (2014), afirma que la forma en que una Spin-off puede permanecer en el tiempo es por medio de obtener licencias de patentes, desarrollar proyectos de investigación y desarrollo (I + D) que fortalezcan el hilo empresarial.

En Venezuela, el apoyo para generar transferencia tecnológica con el modelo Spin-off se basa en los emprendedores y sus particularidades, vincula de una parte a los estudiantes, egresados y profesorado de las universidades, como también a los mecanismos y agentes que convergen para que surja un proyecto de negocio. Se tiene una baja cultura de emprenderismo, lo cual se hace necesario fortalecer como marco de un escenario donde se involucren las Spin-off y los diferentes agentes de su entorno, para formar un tejido empresarial universitario (Cervilla, 2008).

Cuadro 5

Estudios realizados en América Latina

\begin{tabular}{|l|l|l|l|l|l|}
\hline $\begin{array}{l}\text { Base de } \\
\text { datos }\end{array}$ & \multicolumn{1}{|c|}{ País } & \multicolumn{1}{|c|}{ Autor } & Año & \multicolumn{1}{|c|}{ Titulo } & \multicolumn{1}{c|}{ Descripción principal } \\
\hline $\begin{array}{l}\text { Google } \\
\text { Académico }\end{array}$ & Brasil & $\begin{array}{l}\text { Dias y } \\
\text { Porto }\end{array}$ & 2014 & Como a USP transfere tecnologia? & $\begin{array}{l}\text { Regulación en América Latina } \\
\text { para la creación de Spin-off }\end{array}$ \\
\hline WOS & Alemania & $\begin{array}{l}\text { Helm \& } \\
\text { Mauroner }\end{array}$ & 2007 & $\begin{array}{l}\text { Success of Research Based Spin- } \\
\text { offs. State of the art and Guidelines } \\
\text { for Further Research. }\end{array}$ & $\begin{array}{l}\text { Valoración del entorno y } \\
\text { enfoques principales de las } \\
\text { Spin-off en Costa Rica. }\end{array}$ \\
\hline $\begin{array}{l}\text { Google } \\
\text { Académico }\end{array}$ & Costa Rica & $\begin{array}{l}\text { Monge } \\
\text { Agüero }\end{array}$ & 2015 & $\begin{array}{l}\text { Elementos para el diseño de una } \\
\text { estrategia que promueva la } \\
\text { creación de Spin-offs académicas } \\
\text { en las universidades públicas } \\
\text { costarricenses }\end{array}$ & $\begin{array}{l}\text { Factores que promueven la } \\
\text { creación de Spin-off exitosas } \\
\text { en Costa Rica }\end{array}$ \\
\hline DIALNET & Costa Rica & $\begin{array}{l}\text { Monge } \\
\text { Agüero }\end{array}$ & 2018 & $\begin{array}{l}\text { Factores asociados al éxito } \\
\text { competitivo en las Spin-off } \\
\text { académicas de las universidades } \\
\text { públicas costarricenses }\end{array}$ & $\begin{array}{l}\text { Factores que promueven la } \\
\text { creación de Spin-off exitosas } \\
\text { en Costa Rica }\end{array}$ \\
\hline
\end{tabular}




\begin{tabular}{|l|l|l|l|l|l|}
\hline $\begin{array}{c}\text { Base de } \\
\text { datos }\end{array}$ & \multicolumn{1}{|c|}{ País } & \multicolumn{1}{|c|}{ Autor } & Año & \multicolumn{1}{|c|}{ Titulo } & \multicolumn{1}{|c|}{ Descripción principal } \\
\hline DIALNET & México & Feria Patio & 2009 & $\begin{array}{l}\text { Propuesta de un modelo de } \\
\text { transferencia de conocimiento } \\
\text { científico-Tecnológico para México }\end{array}$ & $\begin{array}{l}\text { El entorno y su relación con el } \\
\text { desarrollo de las Spin-off por } \\
\text { medio del conocimiento y el } \\
\text { aprendizaje. }\end{array}$ \\
\hline SCIELO & Argentina & $\begin{array}{l}\text { Malizia, } \\
\text { Sánchez et } \\
\text { al }\end{array}$ & 2013 & $\begin{array}{l}\text { Análisis de los Mecanismos de } \\
\text { Transferencia Tecnológica entre los } \\
\text { Sectores }\end{array}$ & $\begin{array}{l}\text { Evolución y fortalecimiento de } \\
\text { las Spin-off caso Argentina }\end{array}$ \\
\hline SCOPUS & Venezuela & $\begin{array}{l}\text { Cervilla, } \\
\text { M.A }\end{array}$ & 2008 & & $\begin{array}{l}\text { Factores que promueven la } \\
\text { creación de Spin-off exitosas } \\
\text { en Venezuela. }\end{array}$ \\
\hline
\end{tabular}

Fuente: Elaborado por los autores del presente estudio

\subsubsection{Transferencia tecnológica por medio de Spin-off en Colombia}

La evolución de las empresas Spin-off en Colombia se han venido fortaleciendo gracias a las investigaciones que se han desarrollado en los últimos años, los cuales han servido de referencia para poder indagar, sobre sus inicios, regulaciones, normatividad que se han tenido en cuenta para este tipo de modelo, la primera Spin-off en Colombia que ha tenido éxito en su funcionamiento y la relación que debe existir entre universidad- empresa y gobierno, fundamental para la creación de las Spin-off (ver Cuadro 6). A continuación, se expondrá cada una de estas investigaciones con sus hallazgos más importantes.

Las empresas Spin-off son relativamente nuevas en Colombia, se han venido consolidando hace más de diez años por medio de la estrategia dirigida a alcanzar la misión institucional, en los diferentes ámbitos de la universidad, dándole importancia a las investigaciones desarrolladas dentro de estas, teniendo como objetivo la apropiación y extensión del conocimiento hacia la sociedad. En la actualidad, Colombia cuenta con la ley 215 de 2015, la cual promueve el emprendimiento e innovación para dar valor agregado a las IES, se pretende que por medio de las investigaciones se pueda crear empresas de base tecnológica (EBT) por medio Spin-off, que da vida a una nueva unidad productiva (López, 2017). CONOSER,es la primera empresa Spin-off creada en Colombia, ha tenido gran éxito y se encuentra en la universidad de Antioquia, Botero, Giraldo, Castro, Perdomo Charry, y Gómez, 2014; Correa-García, Arango-Serna, y Álvarez-Uribe (2012) afirman que:

El Proyecto de Spin-off Universitaria- CONOSER-Conocimiento y Servicios de Ingeniería surge del grupo de investigación Manejo Eficiente de la Energía (GIMEL) del Departamento de Ingeniería Eléctrica de la Universidad de Antioquia. CONOSER es una empresa prestadora de servicios de consultoría profesional especializada en sistemas eléctricos que, además, ofrece el diseño de equipos para implementar técnicas de mantenimiento predictivo eléctrico en motores de inducción y presta servicios asociados a estos equipos. Actualmente se encuentra en la consolidación de su modelo de negocio, el cual se complementa con el constante desarrollo como grupo de investigación p. 9.

De acuerdo a Gómez (2019) y Bedoya, Behaine, Severiche, Marrugo y Catro ( 2018) afirman que en Colombia dentro de las IES se deben fortalecer aspectos claves para que haya emprendimiento de base tecnológico que ayuden a que las Spin-off se desarrollen, fortaleciendo el hilo empresarial, tales aspectos tienen que ver con: normatividad que evidencie con claridad los derechos patrimoniales y propiedad intelectual, de tal forma que evite el conflicto de intereses entre investigadores, docentes y personal administrativo. También, es fundamental que las IES cuenten con recursos para la investigación y desarrollo (I+D), contar con "capital de riesgo propio", fortalecer el emprendimiento como cultura y contar con programas que lo respalden, saber y conocer el riesgo que se corre al realizar este tipo de transferencia tecnológica y por último, la relación que debe existir entre los actores debe ser excelente. 
En el año 2017, la normatividad colombiana se hizo sentir en este tema por medio de la Ley 1838 de Spin-off "Por la cual se dictan normas de fomento a la ciencia, tecnología e innovación mediante la creación de empresas de base tecnológica (SPIN-OFF) y se dictan otras disposiciones" (Ley 1838, 2017). Es allí, donde las IES, tanto públicas como privadas, son habilitadas para la creación de empresas con base tecnológica, la innovación y el emprendimiento como mecanismos para generar valor agregado en las IES. Su objetivo principal es crear desarrollo económico, humano, de investigación y cultural a nivel regional y nacional, por medio de la transferencia tecnológica.

Con base a la investigación realizada por Gómez (2019) las plataformas e instrumentos con que cuentan las IES para el desarrollo de una Spin-off, son las convocatorias internas y con recursos propios, luego se apalanca con programas tales como Ruta n, Innpulsa, Tecnoova, Colciencias, entre otros; otras IES, se apalancan con inversiones que quieran una titularidad en las Spin-off en los derechos de explotación y patrimoniales.

\section{Cuadro 6}

Estudios realizados en Colombia

\begin{tabular}{|c|c|c|c|c|}
\hline $\begin{array}{c}\text { Base de } \\
\text { datos }\end{array}$ & Autor & Año & Titulo & Descripción principal \\
\hline SCIELO & $\begin{array}{l}\text { López } \\
\text { Obando }\end{array}$ & 2017 & $\begin{array}{l}\text { Surgimiento de empresas catalogados como } \\
\text { Spin-off universitarias en Colombia, análisis } \\
\text { desde la gerencia de proyectos fase I }\end{array}$ & $\begin{array}{l}\text { Inicios de Spin-off en Colombia, } \\
\text { regulaciones que favorecen estas } \\
\text { empresas }\end{array}$ \\
\hline DIALNET & Botero et al & 2014 & $\begin{array}{l}\text { Implementación de Spin-off en el área } \\
\text { informática en Colombia }\end{array}$ & Primera Spin-off exitosa Spin-off \\
\hline SCIELO & $\begin{array}{l}\text { Correa- } \\
\text { García et al }\end{array}$ & 2012 & $\begin{array}{l}\text { Metodología de valoración para proyectos } \\
\text { de transferencia tecnológica universitaria. } \\
\text { Caso aplicado - Universidad de Antioquia }\end{array}$ & $\begin{array}{l}\text { Spin-off en la Universidad } \\
\text { Antioquia y su modelo de negocio }\end{array}$ \\
\hline SCIELO & Gómez & 2019 & $\begin{array}{l}\text { Emprendimiento de Base Tecnológica: Un } \\
\text { reto por cumplir }\end{array}$ & $\begin{array}{l}\text { Normatividad, propiedad } \\
\text { intelectual y plataformas que } \\
\text { deben tener en cuenta las } \\
\text { empresas Spin-off en Colombia }\end{array}$ \\
\hline SCOPUS & $\begin{array}{l}\text { Bedoya, } \\
\text { E.A.; } \\
\text { Behaine, B.; }\end{array}$ & 2018 & $\begin{array}{l}\text { Redes de Conocimiento: Academia, Empresa } \\
\text { y Estado }\end{array}$ & $\begin{array}{l}\text { Integración que debe existir en la } \\
\text { triple Helice. }\end{array}$ \\
\hline $\begin{array}{l}\text { DIARIO } \\
\text { OFICIAL }\end{array}$ & $\begin{array}{l}\text { Congreso de } \\
\text { la Republica } \\
\text { de Colombia }\end{array}$ & 2017 & $\begin{array}{l}\text { Ley } 1838 \text {. Fomento de la Ciencia, la } \\
\text { Tecnología y la innovación, por medio de la } \\
\text { creación de empresas de base tecnológica } \\
\text { (Spin-off) }\end{array}$ & $\begin{array}{l}\text { Regulación en Colombia para las } \\
\text { empresas con base tecnológica } \\
\text { en Spin-off. }\end{array}$ \\
\hline
\end{tabular}

\section{Conclusiones}

A nivel teórico e histórico, los autores estudiados de una u otra forma mencionan a la triple hélice cuando explican el modelo Spin-off. Se ratifica entonces que la efectividad del modelo Spin-off como forma de transferencia tecnológica se fundamenta en el modelo triple hélice, que, en últimas, representa la integralidad y unidad de dirección de un territorio hacia la gestión del conocimiento y que se logra a través de la sinergia de sus principales actores. De una parte, la academia crea conocimiento y busca la forma de transferirlo, el Estado define políticas públicas para orientar el quehacer de los territorios y la industria a través de su dinámica satisface las necesidades y requerimientos del entorno, todos ellos funcionando como un solo cuerpo, al servicio de la sociedad.

A nivel mundial, se reconoce la hegemonía de las empresas Spin-off como uno de los mecanismos más efectivos para realizar transferencia tecnológica, escenario que ha motivado y a la vez ha sido producto, de políticas 
públicas que fomentan la innovación, el desarrollo tecnológico y el emprendimiento. En este sentido, a través del abordaje teórico realizado, se confirma que la educación y, en consecuencia, el conocimiento, es el principal activo de la sociedad y de un territorio; porque es a partir del conocimiento, y de su gestión, como se logran cambios a partir de la innovación que repercuten en el mejoramiento de las condiciones sociales, económicas y empresariales para generar desarrollo, competitividad y mejores condiciones de vida.

Teóricamente, las empresas Spin-off, cuentan con variadas formas de creación, de financiación, de estructura, de operación y de modelo de negocio, así como diferentes escenarios de apoyo, que flexibilizan y en cierto punto facilitan su creación, sin embargo, lo realmente decisivo es la capacidad creativa, innovadora y emprendedora de las personas que las gestan, porque sólo ellas puedan hacerla exitosa, es decir, sostenible.

La evolución de las empresas Spin-off en Norteamérica y Europa demuestran que son un modelo a seguir, en América Latina ha dado grandes frutos en países como Costa Rica y México, la mayor carencia en países de Latinoamérica es la falta de políticas gubernamentales que fomenten y apoyen las ideas innovadoras surgidas desde la universidad para crear empresas con base tecnológica, En Colombia en comparación con Costa Rica y México se requiere mayor financiamiento público e impulso a la generación de conocimiento validado por investigación e innovación; al igual que en México se debe dar más importancia a los centros de investigación. Dentro de los países con menos éxito en Spin-off, se encuentran España, Portugal, Argentina, Venezuela y Colombia, se explica en la falta de ayuda gubernamental, poca legislación, escasa actitud emprendedora de los investigadores y la innovación se considera en un segundo plano.

Si bien, el tema de transferencia de conocimiento es pertinente y atractivo para la investigación, se requiere ahondar en variables que lo pudieran explicar de manera más amplia, por ejemplo: el papel de la educación en la formación de personas creativas, innovadoras y emprendedores en niveles inferiores a la educación superior, que sustente cambios en la sociedad a partir de la gestión del conocimiento; la medición del impacto económico y social de las Spin-off, modelos de estrategia y estructura de las IES para promover transferencia del conocimiento, modelos de negocio para la industria enfocados en este modelo Spin-off y efectividad de las políticas públicas que apalancan el tema, entre otros.

\section{Referencias bibliográficas}

Alavy, M. \& Leidner, D. E., (2001). Review: Knowledge Management and Knowledge Management Systems: Conceptual Foundations and Research Issues. MIS Quarterly, 25(1), pp. 107-136.

Algieri, B., Aquino, A y Succurro, M. (2013). Technology transfer offices and academic Spin-off creation: the case of Italy. J Technol Transf. 38,382-400.

Bedoya, E.A.; Behaine, B.; Severiche, C.A.; Marrugo, Y. y Catro A.F. ( 2018). Redes de Conocimiento: Academia, Empresa y Estado. Revista Espacios. 39(8).

Beraza, J. M. y Rodríguez, A., (2012). Tipología de las Spin-offs en un contexto universitario: Una propuesta de clasificación. Cuadernos de Gestión. 12(1), pp. 39-57.

Botero, R., Giraldo, J. C., Castro, C. A., Perdomo Charry, W., \& Goméz, S. (2014). IMPLEMENTACIÓN DE SPINOFF EN EL ÁREA DE INFORMÁTICA EN COLOMBIA. Ing. USBMed, 5(2), 12-17.

Breznitz, S., O'Shea, R., Allen, T., (2008). University commercialization strategies in the development of regional bioclusters. Journal of Product Innovation Management 25, 129-142.

Cervilla, M.A. (2008). El caso de Celulab: un "Spin-off" de Technoclinical Solutions, empresa incubada en el Parque Tecnológico Sartenejas. Revista Espacios. 29(1). 
Castillo, M., \& Alvarez, A. (2015). La transferencia de investigación en instituciones de educación superior mediante Spin-off. Actualidades investigativas en educación, 15(3), 1-23.

Chang, H. G., (2010). El modelo de triple hélice como un medio para la vinculación entre la universidad y la empresa. Revista Nacional de Administración, 1(1), pp. 85-94.

Clarysse, B., Wright, M., \& Van de Velde, E. (2011). Entrepreneurial origin, technological knowledge, and the growth of spin-off companies. Journal of Management Studies, 48(6), 1420-1442.

Colombia, Congreso de la República. (2017, 6 julio) Ley 1838. Fomento de la Ciencia, la Tecnología y la innovación, por medio de la creación de empresas de base tecnológica (Spin-off). Diario Oficial.

Correa-García, J. A., Arango-Serna, M. D., \& Álvarez-Uribe, K. C. (2012). Metodología de valoración para proyectos de transferencia tecnológica universitaria. Caso aplicado - Universidad de Antioquia. Revista de la Facultad de Ciencias Económicas de la Universidad Militar Nueva Granada. rev.fac.cienc.econ, 10(1), 92106

Dias, A., \& Porto, G. (2014). Como a USP transfere tecnologia?. Organizações \& Sociedade, 21(70).

Etzkowitz, H. \& Peters, L. S., (1990). University-Industry Connections And Academic Values. Technology in Society, 12, pp. 427-440.

Etzkowitz, H. \& Leydesdorff, L., (2000). The dynamics of innovation: from National Systems and "Mode 2" to a Triple Helix of university-industry-government relations. Research Policy, 29, pp. 109-123.

Etzkowitz, H. \& Leydesdorff, L., (1995). The Triple Helix University-Industry-Government relations: A laboratory for knowledge based economic develepment. EASST Review, 14(1), pp. 14-19.

Etzkowitz, H. \& Leydesdorff, L., (1996). Emergence of a Triple Helix of University-Industry-Government. Science and Public Policy, 23, pp. 279-286.

Etzkowitz, H., (1996). The triple helix: Academic-Industry-Government. Annals of the New York Academy of Sciences, 787, pp. 67-86.

Etzkowitz, H. (2013). Anatomy of the entrepreneurial university. Studies of science.52(3).486-511.

Etzkowitz, H. \& Zhou, C., (2018). The Triple Helix: University-Industry-Government. Oxon(New York): Routledge.

Feria, V. H. (2009). Propuesta de un modelo de transferencia de conocimiento científico-tecnológico para México. Universidad Politécnica de Valencia, Valencia.

Fernández-López, S., Pérez-Astray, B., Rodeiro-Pazos, D. and Calvo, N. (2015). Are firms interested in collaborating with universities? An open-innovation perspective in countries of the south of west European space, Service Business, 9(4),637-662.

Fini, R., Grimaldi, R., Santoni, S., \& Sobrero, M. (2011). Complements or substitutes? The role of universities and local context in supporting the creation of academic Spin-offs. Research Policy, 40(8), 1113-1127.

Fong-Reynoso, C. (2010). Transferencia de Conocimiento de la Universidad a la Empresa: La Creación de Empresas Spin-off de Origen Universitario. Sistemas, Cibernética e informática, 7(1), 81-88.

Geuna, A. y Muscio, A (2009). The Governance of University Knowledge Transfer: A Critical Review of the Literature. Minerva. 47,93-114. 
Geuna, A. y Rossi, F. (2011). Changes to university IPR regulations in Europe and the impacto on academic patenting. Research Policy,40,1068-1076.

Giha, Y., Olaya, A., Zapata, L., M., Pontón, J., Rojas, A., Franco, A., López, A., F., Mejía, J., D., Trujillo, A., Betancur, M., C. (2016). Hacía una hoja de ruta para la creación de Spin-off universitarias en Colombia. Medellín: Vallejo Editores Gómez, J. M., Mira, I. \& Verdú, A. J., (2007). Las Spin-offs académicas como vías de transferencia tecnológica. Economía Industrial, Issue 366, pp. 61-72.

Gómez, E. (2019). Emprendimiento de Base Tecnológica: Un reto por cumplir. TEC Empresarial, 13(2), 33-44

Gómez, V., Galeano, H., C., \& Jaramillo, M., D. A. (2015). El estado del arte: Una metodología de investigación. Revista Colombiana De Ciencias Sociales, 6(2), 423-442.

Gómez, J., Mira, I., Verdú, A. y Sancho, J. (2007). Las Spin-off como vía de transferencia tecnológica. Economía Industrial, v. 366, Issue 11, p. 61-72.

González de la Fe, T., (2009). El modelo de triple hélice de relaciones universidad, industria y gobierno: un análisis crítico. ARBOR Ciencia, Pensamiento Y Cultura, pp. 739-755.

Gupte, M. (2007). Success of University Spin-offs: Network Activities and Moderating Effects of Internal Communication and Adhocracy. Wiesbaden: Deutscher Universitäts-Verlag.

Hanel, P. and St-Pierre, M. (2006). Industry-university collaboration by Canadian manufacturing firms, Journal of Technology Transfer,31(4),485-499.

Helm, R., \& Mauroner, O. (2007). Success of Research Based Spin-offs. State of the art and Guidelines for Further Research. Review of Managerial Science, 1(3), 237-270

Huyghe, A., Knockaert, M., Wright, M. \& Piva, E., (2014). Technology transfer offices as boundary spanners in the pre-Spin-off procces: The case of a hybrid model. Small Business Economics, 43(2), pp. 289-307.

Landry, R., Amara, N., \& Rherrad, I. (2006). Why are some university researchers more likely to create Spin-offs than others? Evidence from Canadian universities. Research Policy, 35(10), 1599-1615.

Leydesdorff, L. (2012). The Triple Helix, Quadruple Helix, ..., and an N-Tuple of Helices: Explanatory Models For Analyzing The Knowledge-Based Economy?. Journal of the Knowledge Economy, 3(1), pp. 25-35.

Lawton-Smith, H., y Ho, K. (2006). Measuring the performance of Oxford University, Oxford Brookes University and the government laboratories Spin-off companies. Research Policy, 35(10), 1554-1568.

Lockett, A., Siegel, D., Wright, M., \& Ensley, M. (2005). The creation of Spin-off firms at public research institutions: managerial and policy implications. Resource Policy, 34(7), 981-993.

López, P. (2017). Surgimiento de empresas catalogadas como Spin-off universitarias en Colombia, análisis desde la gerencia de proyectos fase I. EAN, 61-72.

Malizia, A. I., Sánchez Barrioluengo, M., Lombera, G., \& Castro Martínez, E. (2013). Análisis de los Mecanismos de Transferencia Tecnológica entre los Sectores. Journal of Technology Management \& Innovation, 103115.

Martínez-Gómez, J., (2017). Procesos de transferencia de conocimiento en una Facultad de Ciencias Contables. Revista Espacios, 38(50), pp. 1-7. 
Marulanda, C. E. \& López, M., (2013). La gestión del conocimiento en las PYMES de Colombia. Revista Virtual Universidad Católica Del Norte, Febrero-Mayo, Issue 38, p. 13.

Meisel D., C. A., Bermeo, H. P., \& Oviedo Muñoz, L. (2006). Generación de valor a través de la gestión estratégica del conocimiento, de la innovación y la mejora continua "un modelo de aplicación al sistema de operaciones de una pyme manufacturera". Scientia et Technica, 12(31), 165-170.

Miller, K., McAdam, R., Moffett, S. \& Brennan, M., (2011). An exploratory study of retaining and maintining knowledge in university technology transfer proccesses. Internationla Journal Of Entrepreneurial Behavior And Research, 17(6), pp. 663-684.

Monge-A, M. (2018). Factores asociados al éxito competitivo en las Spin-off académicas de las universidades públicas costarricenses. Small Business International Review, 2(2), 54-70.

Monge-A, M., Briones-Peñalver, A. J., \& Lema, D. G.-P. d. (2015). Elementos para el diseño de una estrategia que promueva la creación de Spin-offs académicas en las universidades públicas costarricenses. Tecnología en Marcha, 29(2), 25-42.

Monge-A., M., Briones-P., A.J. y García-P. de Lema, D. (2011). Factores determinantes de la creación de las Spin-off académicas: caso del Instituto Tecnológico de Costa Rica. Cuadernos de Administración, 27(46).

Morales, M., Pineda, K., \& Ávila, K. (2012). Organizaciones innovadoras a partir de la interacción con la universidad: casos exitosos. Estudios Gerenciales, 8, 363-374.

Nonaka, I., (1994). A Dynamic Theory of Organizational Knowledge Creation. Oraganization Science , 5(1), pp. 14-37.

Olmos-Peñuela, J., Castro-Martinez, E. \& D'este Cukierman, P., (2014). Actividades de transferencia de conocimiento en ciencias sociales y humanidades: Explicación de las interacciones de grupos de investigación con agentes no académicos. Research Policy, 43(4), pp. 696-706.

O'shea, R. P., Allen, T. J., Chevalier, A., \& Roche, F. (2005). Entrepreneurial orientation, technology transfer and spinoff performance of US universities. Research policy, 34(7), 994-1009.

Rasmussen, E. (2008). Government instruments to support the commercialization of university research: lessons from Canada. Technovation, 28(8), 506-517.

Rasmussen, E., Mosey, S., Wright, M. (2013a). The Evolution of Entrepreneurial Competencies: A Longitudinal Study of University Spin-off Venture Emergencejoms. Journal of Management Studies, 48(6).1314-1345

Rasmussen, E., Mosey, S., Wright, M. (2013b). The influence of university departments on the evolution of entrepreneurial competencies in Spin-off Ventures. Research policy,43(2014),92-106.

Robertson, J., McCarthy, I. P. \& Pitt, L., (2019). Leveraging social capital in university-industry knowledge transfer strategies: a comparative positioning framework. Knowledge Management Research \& Practice, 17(4), pp. 461-472.

Rodeiro-Pazos, D., Fernández-López, S., Otero-González, L. and Rodríguez-Sandiás, A. (2012) A resource-based view of university Spin-off activity: new evidence from the Spanish case, Revista Europea de Dirección y Economía de la Empresa, 21(3), 255-265.

Sautu, R., Boniolo, P., Dalle, P., \& Elbert, R. (2005). Manual De Metodología. Construcción del marco, formulación de los objetivos y elección de la metodología. Buenos Aires: CLACSO. 
Segarra C., M., \% Bou LI., J. C. (2005). Concepto, tipos y dimensiones del conocimiento: ConFiguración del conocimiento estratégico. Revista De Economía Y Empresa, (53), 175-195.

¡ Tarrés, C. (2008). Transferencia Tecnológica. Empresa - Universidad: La nueva visión de las universidades. DYNA, (Vol. 83).

Sierra, J. (2018). La cuarta hélice y la financiación de la innovación. Journal Of Economics, Finance And Administrative Science, 23(45), pp. 128-137.

Stankiewicz, R., (1994). Spin-off companies from universities. Science And Public Policy, 21(2), pp. 99-107.

Teece, D. J., Pisano, G. \& Shuen, A., (1997). Dynamic Capabilities And Strategic Management. Strategic Management Journal, 18(7), pp. 509-533.

Touriñán, J. M. (2019). La transferencia de conocimiento como proceso: de la universidad al sector educativo. Una mirada desde la pedagogía. Boletín Repide, 8(3), 19-65.

Valmaseda, O., \& Hernández, N. (2012). Fuentes de conocimiento en los procesos de innovación empresarial: las Spin-off universitarias en Andalucía. ARBOR Ciencia, Pensamiento y Cultura, 188(753).

Visintin, F y Pittino, D. (2013). Founding team composition and early performance of university-Based Spin-off companies. Technovation. 34(2014), 31-43

Esta obra está bajo una Licencia Creative Commons Attribución-NoCommercial 4.0 International

(cc) BY-NC 